\title{
Landau Theory of Stripe Phases in Cuprates and Nickelates
}

\author{
Oron Zachar, S. A. Kivelson \\ Dept. of Physics, UCLA Los Angeles, CA 90095-1547 \\ V. J. Emery \\ Dept. of Physics, Brookhaven National Laboratory, Upton, NY 11973-5000
}

(February 27, 2018)

\begin{abstract}
We consider a Landau theory of coupled charge and spin-density wave order parameters as a simple model for the ordering that has been observed experimentally in the $\mathrm{La}_{2} \mathrm{NiO}_{4}$ and $\mathrm{La}_{2} \mathrm{CuO}_{4}$ families of doped antiferromagnets. The period of the charge-density wave is generically half that of the spin-density wave, or equivalently the charges form antiphase domain walls in the antiferromagnetic order. A sharp distinction exists between the case in which the ordering is primarily charge driven (which produces a sequence of transitions in qualitative agreement with experiment) or spin driven (which does not). We also find that stripes with non-collinear spin order (i.e. spiral phases) are possible in a region of the phase diagram where the transition is spin driven; the spiral is circular only when there is no charge order, and is otherwise elliptical with an eccentricity proportional to the magnitude of the charge order.
\end{abstract}

Experiments on the doped lanthanum nickelate [1] and lanthanum cuprate 22.53 families of materials have established that topological stripe phases are a prominent feature of doped antiferromagnets. In the low-temperature stripe phase, the doped holes are concentrated in periodic walls which are simultaneously discommensurations in the Néel order. In this paper, the phase diagram of coupled charge density wave (CDW) and spin density wave (SDW) order parameters will be constructed from the Landau theory of phase transitions [4]. By associating the experimental observations with distinct regions of the global phase diagram it is possible to gain insight into the microscopic mechanism of stripe formation and the nature of the spin ordering. It will be shown that the experiments are consistent with the suggestion that stripes are produced by frustrated phase separation [5 5 and not by a Fermi surface instability [8].

The analysis also addresses the existence of spiral magnetic order found in some theoretical studies of doped antiferromagnets [9]. A circular spiral phase (in which the magnitude of the ordered moment is a constant) is only possible if there is no accompanying charge order; the coupling of the spin and charge order generally produces an elliptical spiral phase, with the eccentricity of the ellipse proportional to the magnitude of the charge order parameter. In the region of the phase diagram which we associate with all known experiments, spiral phases are a remote possibility. They may appear only as a third transition (yet undetected) at very low temperatures.

It will be assumed that the Landau free energy function depends on only the fundamental Fourier components of the SDW and CDW order parameters. Of course, as always, higher harmonics will appear as the magnitude of the order increases, but this has no effect on the nature of the phase diagram. So as to focus on the situation of immediate experimental relevance, we will consider the quasi-two dimensional case in which the ordering vectors lie in a plane (and, ultimately, in a line), but the generalization to other geometries is straightforward. (We shall return to the issue of the fluctuation effects peculiar to quasi-two-dimensional systems at the end.) Also, we consider the case in which the crystal has the symmetry of a square lattice, and the ordering vectors lie along a symmetry direction, so that there are only two inequivalent directions of the ordering vector (along the diagonals in the case of the lanthanum nickelates, and along the vertical and horizontal directions for the lanthanum cuprates). This will allow us to extract the essential physics in a relatively simple way. The generalizations to the various space groups of the structures of any given material (which in principle should be used) and to allow different ordering vectors is straightforward.

With these restrictions, the stripe order can be described by the two complex scalars, $\rho_{\vec{k}}$ and $\rho_{\vec{k}^{\prime}}$ and the complex spin vectors $\mathbf{S}_{\vec{q}}$ and $\mathbf{S}_{\vec{q}^{\prime}}$, corresponding to charge order and spin order respectively. Here, the vectors $(\vec{k}$, $\vec{q})$ are related to $\left(\vec{k}^{\prime}, \vec{q}^{\prime}\right)$ by rotation through $\pi / 2$, and $\vec{q}$ is measured relative to the magnetic ordering vector $\vec{Q}$ of the undoped system. (It is assumed that $\vec{Q}$ is unique, which requires that $\vec{Q} \equiv-\vec{Q}$, i.e. that $2 \vec{Q}$ must be equal to a reciprocal lattice vector.)

The most general free energy up to fourth order is constructed by including all terms allowed by symmetry, i.e. translation, time reversal, reflection, and spin rotation invariance, and the crystal point-group symmetries:

$$
F=\mathcal{F}\left(\rho_{\vec{k}}, \mathbf{S}_{\vec{q}}\right)+\mathcal{F}\left(\rho_{\vec{k}^{\prime}}, \mathbf{S}_{\vec{q}^{\prime}}\right)+\mathcal{F}_{\text {int }}\left(\rho_{\vec{k}}, \mathbf{S}_{\vec{q}}, \rho_{\vec{k}^{\prime}}, \mathbf{S}_{\vec{q}^{\prime}}\right)
$$




$$
\begin{aligned}
\mathcal{F}= & \frac{1}{2} r_{\rho}|\rho|^{2}+U_{\rho}|\rho|^{4} \\
& +\frac{1}{2} r_{s}|\mathbf{S}|^{2}+U_{s}|\mathbf{S}|^{4}+U_{x}\left(\mathbf{S} \times \mathbf{S}^{*}\right) \cdot\left(\mathbf{S} \times \mathbf{S}^{*}\right) \\
& +\lambda_{1}\left[(\mathbf{S} \cdot \mathbf{S}) \rho^{*}+\text { c.c }\right]+2 \lambda_{2}|\mathbf{S}|^{2}|\rho|^{2} .
\end{aligned}
$$

Note that it is not necessary to include separate contributions from wave vectors $-\vec{q}$ and $-\vec{k}$ since the charge and spin densities are real, and hence $\rho_{-\vec{k}}=\rho_{\vec{k}}^{*}$ and $\mathbf{S}_{-\vec{q}}=\mathbf{S}_{\vec{q}}^{*}$. Also, there is no separate contribution of the form $|\mathbf{S} \cdot \mathbf{S}|^{2}$ because it may be written as a linear combination of the other quartic terms. $\mathcal{F}_{\text {int }}$, which is entirely quartic, has cross terms coupling the order at $(\vec{q}, \vec{k})$ and $\left(\vec{q}^{\prime}, \vec{k}^{\prime}\right)\left(\right.$ e.g. $\left.\left.\quad V_{1}|\mathbf{S}|^{2}|| \mathbf{S}^{\prime}\right|^{2}\right)$. For simplicity, and because it is the case of experimental interest, it will be assumed that the interactions in $\mathcal{F}_{\text {int }}$ are uniformly repulsive, so that unidirectional order is favored (i.e. spin order will occur at $\vec{q}$ or $\vec{q}^{\prime}$, but not both). For example, we do not allow $\mathcal{F}_{\text {int }}$ to contain terms that favor checkboard order [6] as an alternative to stripe order.

The third order term $\left(\lambda_{1}\right)$ coupling spin and charge is allowed if and only if

$$
\vec{k}=2 \vec{q} .
$$

As discussed previously [10], this relation is the generic reason for the "topological" character of the dopantinduced structure, as it implies that the period of the spin modulation is twice the period of the charge modulation or, in other words, a periodic array of hole lines induces an array of antiphase spin domains.

The free energy in Eq. (1) does not contain umklapp terms, in which the sum of wave vectors is equal to a reciprocal lattice vector $\vec{G}$, which become important in the neighborhood of a commensurability. Assuming that Eq. (2) is satisfied, the possible umklapp contributions up to fourth order are $\mathbf{S} \cdot \mathbf{S}$ (when $2 \vec{q}=\vec{G}$ ); $\rho^{2}, \mathbf{S} \cdot \mathbf{S} \rho$, and $(\mathbf{S} \cdot \mathbf{S})^{2}($ all when $4 \vec{q}=G) ; \rho^{3}($ when $6 \vec{q}=G)$ and $\rho^{4}$ (when $8 \vec{q}=G$ ). Higher order terms give (weaker) commensurabilities at smaller wave vectors. As usual [11], the system will display commensurate regions separated by soliton "discommensurations" when the wave vector is close to a commensurate value.

From now on we will drop the subscripts on the order parameters, since each order parameter has a single wave vector, as specified above. Also the normalization of the order parameters will be chosen so that $U_{\rho}=1=U_{s}$.

Nature of the ordered phases: With only two wave vectors $( \pm \vec{q})$, the spin order must be either collinear or coplanar, since a full three dimensional spin texture requires at least three ordering vectors. We consider the collinear and the non-collinear cases separately.

When the spin order is collinear, the axes and origin of coordinates may be chosen so that $\mathbf{S}=|S| \mathbf{e}_{\mathbf{1}}$ and $\rho=|\rho| e^{i \theta}$, where $\theta$ determines the relative phase of the charge and spin density waves. In real space this means that the charge and spin density are

$$
\begin{aligned}
& \rho(\vec{r})-\bar{\rho}=2|\rho| \cos (2 \vec{q} \cdot \vec{r}-\theta), \\
& \mathbf{S}(\vec{r}) e^{i \vec{Q} \cdot \vec{r}}=2|S| \mathbf{e}_{\mathbf{1}} \cos (\overrightarrow{\mathbf{q}} \cdot \overrightarrow{\mathbf{r}}) .
\end{aligned}
$$

It is easily seen that the free energy is minimized with $\theta=\pi$ for $\lambda_{1}>0$ and $\theta=0$ for $\lambda_{1}<0$. Clearly, for $\theta=\pi$, the charge density is peaked on the spin domain walls where the magnitude of the spin order is zero. Since, in all microscopic models studied to date, doping tends to depress magnetic order, we expect on general grounds that $\lambda_{1}>0$.

Thus, for collinear spins, the Landau free energy can be expressed in terms of the magnitudes of the order parameters as

$$
\begin{aligned}
F_{\text {linear }}(|\rho|,|S|) & =\frac{1}{2} r_{s}|S|^{2}+|S|^{4} \\
& +\frac{1}{2} r_{\rho}|\rho|^{2}+|\rho|^{4} \\
& -2\left|\lambda_{1}\right||S|^{2}|\rho|+2 \lambda_{2}|S|^{2}|\rho|^{2} .
\end{aligned}
$$

For a coplanar spiral phase, the origin of coordinates and axis of quantization may be chosen so that $\mathbf{S}=|S|\left[\cos (\alpha) \mathbf{e}_{1} \pm i \sin (\alpha) \mathbf{e}_{2}\right]$, which corresponds to a real-space spin density

$$
\begin{aligned}
& \mathbf{S}(\vec{r}) e^{i \vec{Q} \cdot \vec{r}}= \\
& \quad 2|S|\left[\cos (\alpha) \cos (\vec{q} \cdot \vec{r}) \mathbf{e}_{1} \pm \sin (\alpha) \sin (\vec{q} \cdot \vec{r}) \mathbf{e}_{2}\right] .
\end{aligned}
$$

Clearly, $\tan \alpha$ determines the eccentricity of the elliptical spiral, and $\alpha=\pi / 4$ corresponds to an ideal spi$\mathrm{ral}$, in which the magnitude of the magnetic order is a constant. The minimization of the free energy (with the assumption that $|S| \neq 0$ ) with respect to $\alpha$ (in the range $0<\alpha \leq \pi / 4)$ and $\theta$ can be carried out straightforwardly. If $\left|\lambda_{1}\right||\rho| / U_{x}|S|^{2} \geq 1$, the result is $\alpha=0$, or in other words the collinear state is recovered. For $\left|\lambda_{1}\right||\rho| / U_{x}|S|^{2}<1$, the free energy is minimized for

$$
|S|^{2} \cos (2 \alpha)=\left|S_{1}\right|^{2}-\left|S_{2}\right|^{2}=\left|\lambda_{1}\right||\rho| / U_{x}
$$

and as a function of $|\rho|$ and $|S|$ the free energy of the spiral state is

$$
\begin{aligned}
F_{\text {spiral }}(|\rho|,|S|) & =\frac{1}{2} r_{s}|S|^{2}+\left(1-U_{x}\right)|S|^{4} \\
& +\frac{1}{2}\left(r_{\rho}-\frac{2 \lambda_{1}^{2}}{U_{x}}\right)|\rho|^{2}+|\rho|^{4} \\
& +2 \lambda_{2}|S|^{2}|\rho|^{2} .
\end{aligned}
$$

The spiral phase is limited by the constraint $|\cos (2 \alpha)| \leq$ 1 , which for the simple case of $\lambda_{2}=0$ is satisfied only for

$$
r_{s}^{2}\left[\frac{U_{x}}{2\left|\lambda_{1}\right|\left(1-U_{x}\right)}\right]^{2}+\left(r_{\rho}-\frac{2 \lambda_{1}^{2}}{U_{x}}\right) \geq 0
$$




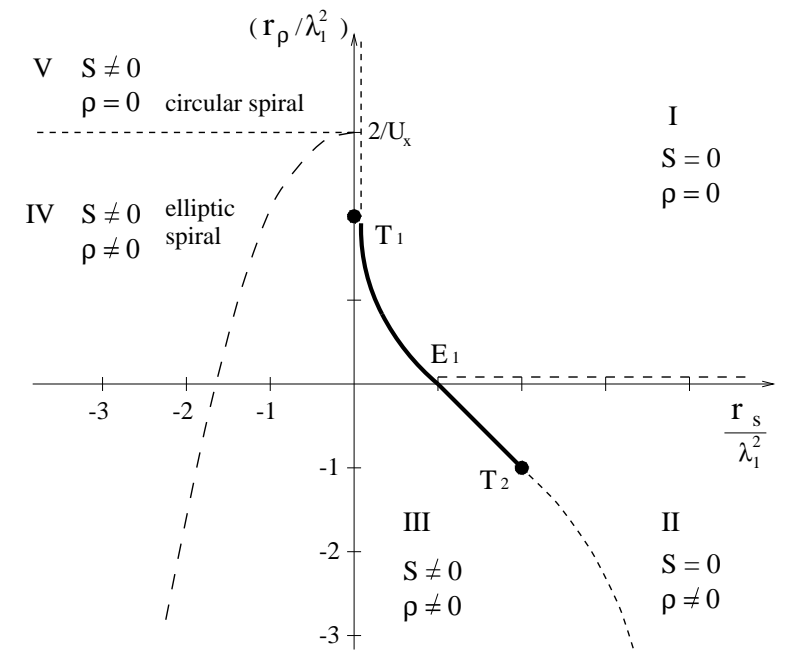

FIG. 1.

The phase diagram shown in Fig. 1 was derived by minimizing the free energy in Eq. (1) with respect to $\rho$ and $\mathbf{S}$ where, to be concrete, we have shown the locations of the phase boundaries for the case $\lambda_{2}=0$ and $0<U_{x}<$ 1. It exhibits five distinct phases: $\mathbf{I})$ a disordered phase, with $|\rho|=0$ and $|S|=0 ; \mathbf{I I}$ ) a charge ordered phase with $|\rho| \neq 0$ and $|S|=0$; III) a collinear stripe ordered phase with $|\rho| \neq 0$ and $|S| \neq 0$; IV) an elliptical-spiral phase, with $|\rho| \neq 0$ and $|S| \neq 0$ and $0<\alpha<\pi / 4$ as determined from Eq. (6). V) a circular spiral phase with spin order but no accompanying charge order. For $U_{x} \leq 0$, the two spiral phases are eliminated from the phase diagram, but the remaining phase boundaries are unchanged.

Let us briefly sketch the analysis that leads to this phase diagram:

The Spin Driven Transition to a Collinear Phase: For $r_{\rho}>0$, minimizing $F_{\text {linear }}$ with respect to $\rho$ gives

$$
|\rho|=\frac{2 \lambda_{1}|S|^{2}}{r_{\rho}}+\mathcal{O}\left(|S|^{4}\right),
$$

and, on eliminating $\rho$ to obtain an effective free energy for the spins,

$$
F_{\text {linear }}^{\text {eff }}(|S|)=\frac{1}{2} r_{s}|S|^{2}+\left(1-\frac{2 \lambda_{1}^{2}}{r_{\rho}}\right)|S|^{4}+\mathcal{O}\left(|S|^{6}\right) .
$$

Clearly, for $r_{\rho}>2 \lambda_{1}^{2}$, there is a second order transition as a function of $r_{s}$ from the disordered phase for $r_{s}>0$ to the collinear stripe phase for $r_{s}<0$. This transition is spin driven; if we assume that $r_{s} \propto\left(T-T_{c}\right)$, we find the usual mean-field exponent, $|S| \sim\left(T_{c}-T\right)^{1 / 2}$, while the charge modulation, which is parasitic to the spin order, grows more slowly, as $|\rho| \sim|S|^{2} \sim\left(T_{c}-T\right)$. The second order line along $r_{s}=0$ ends at a tricritical point, denoted by $\mathrm{T}_{1}$ in Fig. 1 , where $r_{s}=0$ and $r_{\rho}=2 \lambda_{1}^{2}$, so that the coefficients of both the $|S|^{2}$ and $|S|^{4}$ terms in $F^{\text {eff }}(|S|)$ vanish.
The Transition Driven by Spin-Charge Coupling: Below the tricritical point, where $r_{\rho}<2 \lambda_{1}^{2}$, the transition becomes first order, and moves into the quadrant in which both $r_{s}>0$ and $r_{\rho}>0$; here, the transition is driven by the coupling between spin and charge. The precise shape of the first order line depends, in general, on $\lambda_{2}$ and on the values of higher order terms neglected in our truncated form of the Landau free energy in Eq. (1); the general topology and structure of the phase diagram shown in Fig. 1 are, however, unchanged by these higher order terms.

The Charge Driven Transitions: For $r_{s}$ positive and sufficiently large, there is a second order line along the $r_{\rho}=0$ axis separating the disordered and charge-ordered phases. This line ends at a critical end point, $E_{1}$ in the figure, where the first-order line discussed previously crosses the axis. In this range of $r_{s}$, there is a second transition at negative $r_{\rho}$ from the charge-ordered phase to the collinear stripe phase, at which the spin-charge coupling finally causes the spin density to order as well. Again, one can analyze this transition by first minimizing the free energy with respect to $\rho$, and then analyzing $F^{e f f}$ as a function of $S$. As a result, there is a second tricritical point, $T_{2}$, at which this transition changes from first order (an extention of the previously discussed first order line) to second order. In either case, the spin order, enhances the charge order. In this region of the phase diagram, higher order terms in the Landau free energy can have quantatitive ( but, we believe, not qualitative) effects on the results. However, to be concrete, it is useful to display analytic results obtained with $\lambda_{2}$ and all higher order terms neglected. Specifically, in this case,

$$
\rho=\rho_{0}+\left[\lambda_{1} /\left|r_{\rho}\right|\right]|S|^{2}+\mathcal{O}\left(|S|^{4}\right),
$$

where $\rho_{0}=\sqrt{\left|r_{\rho}\right|} / 2$ is the value of $\rho$ in the chargeordered phase, $T_{2}$ is the point $r_{s}=2 \lambda_{1}^{2}$ and $r_{\rho}=-\lambda_{1}^{2}$, and the second order line is given by the expression $r_{s}=2 \lambda_{1} \sqrt{\left|r_{\rho}\right|}$. The first order line, which is given by the expression $r_{s}=\lambda_{1}^{2}+\left|r_{\rho}\right|$, can be located straightforwardly once it is realized that on approaching this line form the stripe-ordered side, $\rho=\lambda_{1} / 2$ and $|S|^{2}=(1 / 4)\left(2 \lambda_{1}^{2}-r_{s}\right)$.

The Transition to the Spiral Phases: For $U_{x}<0$, it is easy to see that a collinear phase always has lower free energy than any competitive spiral phase. For $U_{x}>1$, the Landau free energy as written is unbounded below, so higher order terms must be included in any analysis. However, for $r_{s}<0$ and $0<U_{x}<1$, there is the possiblility of a spiral phase.

Therefore, to complete the phase diagram for $r_{s}<0$ and $0<U_{x}<1$, one must compute the minimal value of $F_{\text {spiral }}$ (subject to the constraint $|\cos (2 \alpha)|=$ $\left.\left|\lambda_{1}\right||\rho| / U_{x}\left|S^{2}\right| \leq 1\right)$ and compare it with the minimal value of $F_{\text {linear }}$. For simplicity, we first consider the case $\lambda_{2}=0$. It is easy then to see that for $r_{\rho} / \lambda_{1}^{2}-2 / U_{x}>0$, $F_{\text {spiral }}$ is minimized by $\rho=0$ and that $F_{\text {spiral }}<F_{\text {linear }}$; this is region $\mathrm{V}$ of the phase diagram, the circular spiralspin phase. At the point where $r_{\rho} / \lambda_{1}^{2}-2 / U_{x}$ changes sign, 
it is clear from Eqs. (6) and (7) that there is a second order transition (from region-V to region-IV ) marked by the onset of both charge order, and an elliptical eccentricity to the spin spiral. As $r_{\rho}$ decreases, the spiral eccentricity gradually increases until it achieves its maximum value (linear polarization). The line $\cos (2 \alpha)=1$ thus determines the phase boundary between regions IV and III of the phase diagram; for $\lambda_{2}=0$, this line is simply the parabola determined by Eq. (8). The main effect of $\lambda_{2}>0$ (in this region of the phase diagram) is to expand the region of the circular spiral phase at the expense of the elliptical spiral phase. For $\lambda_{2}>\sqrt{\left(1-U_{x}\right)}$ the elliptical spiral phase is completely eliminated. Conversely, of course, $\lambda_{2}<0$ tends to stabilize the elliptical spiral phase.

\section{Further Theoretical Considerations:}

Effect of Higher Harmonics: So far we have considered only ordering at the fundamental wave vector, although of course higher harmonics are induced below $T_{c}$. It is important to verify that these harmonics do not affect the stability of the various phases. Slightly below $T_{c}$, in a spin-ordered phase, the effective free energy to order $\left|S_{n \vec{q}}\right|^{2}$ for the $n^{t h}$ harmonic is of the form

$$
\mathcal{F}_{n}=r_{n}\left|\mathbf{S}_{n \vec{q}}\right|^{2}+\left[\mathbf{A}_{n} \cdot \mathbf{S}_{n \vec{q}}+\text { c.c. }\right]
$$

where $r_{n}>0$ and $\mathbf{A}_{n}$ is a function of $\mathbf{S}_{\vec{q}}$ and $\rho_{\vec{k}}$, if there is charge order as well. For a collinear phase, by rotational invariance, $\mathbf{A}_{n} \propto \mathbf{S}$, so the induced higher harmonics are always parallel to the fundamental. Similarly, for a spiral phase with unbroken time-reversal symmetry, it is straightforward to see that $\mathbf{A}_{n}$ must lie in the ordering plane, so that the planar character of this phase is unaffected by higher harmonics. However, if time reversal symmetry is broken, then a contribution to $\mathbf{A}_{0} \propto \mathbf{S}_{\vec{q}} \times \mathbf{S}_{-\vec{q}}$ is allowed and the planar phase is unstable to the formation of a three-dimensional spiral.

Goldstone Modes and Fluctuation Effects in Collinear Phases: Landau theory is, of course, mean-field theory, so it is important to address the effects of fluctuations about the mean-field state. In the absence of commensurability effects, there is a Goldstone mode which reflects the broken translational symmetry associated with finite $\vec{q}$ ordering; commensurability effects, if relevant, will produce a gap in this mode, which will be smaller the higher the order of the commensurability. In any of the collinear spin-ordered phases, there are two Goldstone modes which reflect the broken spin-rotational symmetry. Any uniaxial (Ising) anisotropy would produce a gap in these modes.

Thermal fluctuations of these low lying modes may not dramatically alter the phase diagram in three dimensions, but in quasi-two dimensional systems, such as $\mathrm{La}_{1.6-x} \mathrm{Nd}_{0.4} \mathrm{Sr}_{x} \mathrm{CuO}_{4}$, they always destroy the longrange order, unless terms that break the symmetry (e.g. Ising anisotropy and umklapp scattering ) or inter-plane couplings (i.e. three dimensional effects) are included in the analysis. However, in many cases, this observation is accademic. For instance, the correlation length of the two-dimensional spin $1 / 2$ Heisenberg model is roughly proportional to $\exp (J / T)$ at low temperatures [13] (where $J$ is the exchange integral and $a$ is the lattice constant). Thus the magnetic correlation length can exceed the size of the sample at temperatures of interest and, for all practical purposes, the state of the system is indistinguishable from long-range order. The effects of disorder are also potentially dramatic in two dimensions where the density wave order will generally break up into Lee-Rice domains [14], but, again, if the disorder is sufficiently weak, this may be of largely accademic interest.

Goldstone Modes of the Spiral Phase: The elliptical spiral phase has, in addition to the one Goldstone mode associated with broken translational symmetry, and the two Goldstone modes associated with rotations of the principal axis of the elipse, an additional Goldstone mode associated with rotations about the principal axis. Ising anisotropy will open a gap in two of the spin-related Goldstone modes, but will leave the third one gapless; it requires XYZ anisotropy to fully gap the spin-related Goldstone modes. Thus, the presence of a third Goldstone mode, in particular one with an anomalously small gap, can be used as a diagnostic for the presence of elliptical spin order.

In the circular spiral phase, rotations in the plane of the spiral are equivalent to a translation, so the number of Goldstone modes is the same as in the collinear phase. However, the mode related to translational invariance has a spin component, and thus is relatively insensitive to commensurability effects and to the effects of disorder.

Relation to experimental results: Several features of the experiments on the cuprates and nickelates may be discussed in terms of the Landau theory analysis. Both $\mathrm{La}_{1.6-x} \mathrm{Nd}_{0.4} \mathrm{Sr}_{x} \mathrm{CuO}_{4}$ [2] and doped nickelates [1] show an onset of charge order prior to spin order. $\mathrm{La}_{1.6-x} \mathrm{Nd}_{0.4} \mathrm{Sr}_{x} \mathrm{CuO}_{4}$ undergoes two continuous transitions (onset of charge order and later spin order), and therefore, can be associated with a path on the phase diagram that goes from phase-I to phase-II, and then to phase-III through second order transitions. Several $\mathrm{La}_{2} \mathrm{NiO}_{4+\delta}$ samples [1] clearly show a first order transition associated with the onset of spin order in the transition from phase-II $(|\rho| \neq 0,|S|=0)$ to the full stripe order, phase-III. Thus, these experiments can be associated with a path on the phase diagram that goes through the first order transition line between point $E_{1}$ and the tricritical point $T_{2}$. In both cases, it is clear that the transitions are charge driven rather than spin driven. Note that in this region of the phase diagram there is very little prospect of having a third phase transition to a spiral state, unless it occurs at much lower temperatures.

The above discussion has said nothing about the particular value of the wave vector $k=2 q$. In the Landau theory, the value of $q$ can only depend on the (nonspecific) $q$-dependence of the coefficients of the various terms in the free energy, or on commensurability. Experimentally, in the cuprates [2,3], the ordered stripes 
in $\mathrm{La}_{1.6-x} \mathrm{Nd}_{0.4} \mathrm{Sr}_{x} \mathrm{CuO}_{4}$ and the fluctuating stripes in $\mathrm{La}_{2-x} \mathrm{Sr}_{x} \mathrm{CuO}_{4}$ have $q \approx x$ for $x<1 / 8$, which corresponds to one hole per two $\mathrm{Cu}$ along the stripe. For $x>1 / 8, q \approx 1 / 8$ in $\mathrm{La}_{2-x} \mathrm{Sr}_{x} \mathrm{CuO}_{4}$ and somewhat larger in $\mathrm{La}_{1.6-x} \mathrm{Nd}_{0.4} \mathrm{Sr}_{x} \mathrm{CuO}_{4}$. (Here, $q$ is measured in units of $2 \pi / a$.) This suggests that the value of $q$ is influenced by the internal structure of the stripe for $x<1 / 8$, and by the $\rho^{4}$ umklapp term for $x>1 / 8$. Furthermore, the temperature dependence of $q$ in the ordered phase of $\mathrm{La}_{1.6-x} \mathrm{Nd}_{0.4} \mathrm{Sr}_{x} \mathrm{CuO}_{4}$ is not strong, which suggests that the amplitude of the stripe is well established at the ordering temperature, and that the transition is produced by phase ordering, as expected for quasi two-dimensional systems. In all respects, the value of the stripe wave vector $q$ is determined by charge dynamics, rather than spin dynamics.

The conclusion of this analysis that stripes are charge driven rather than spin driven supports the idea that the driving force is Coulomb-frustrated phase separation [5.6 driven by the hole dynamics. In order to minimize their kinetic energy, the holes attempt to separate into hole-rich regions and regions with significant local antiferromagnetic correlations. However phase separation is frustrated by the long-range Coulomb interaction between the holes, and the compromise between these two forces is the (charge-driven) stripe phase. The antiphase ordering of the spin domains is a consequence of transverse stripe fluctuations; it ensures that, on average, adjacent spins are antiparallel, whatever the location of the stripe.

The alternative mechanism for stripe formation is a Fermi surface instability [8] (due to nesting), in which the spins form antiphase domains that are stabilized by holes bound to the domain walls. In this scenario, spin and charge order together or charge stripe order follows after spin order, contrary to experiment. These microscopic theories also predicted the possibility of spiral phases [9] (though not that they must be elliptical). We conclude that theories based on a Fermi surface instability may be relevant in the spin-driven region of the phase diagram $\left(r_{\rho}>0\right)$ but not for any of the materials of interest, which all show a charge driven sequence of transitions.

Indeed there are more general reasons to believe that the ordered phases in the high- $\mathrm{T}_{c}$ cuprates should not arise from a Fermi surface instability. It has been argued [12] that these materials belong to a class of "bad metals", in which there are no quasiparicles, and therefore no Fermi surface or Fermi surface instabilities.

Acknowledgements: We thank John Tranquada for numerous discussions of his experimental results on the cuprates and nickelates, and especially the implications of the Landau theory. This work was supported in part by the National Science Foundation grant number DMR93-12606 (SK \& OZ) at UCLA. Work at Brookhaven (VE) was supported by the Division of Materials Science, U. S. Department of Energy under contract No. DE-AC02-76CH00016.
[1] C. H. Chen, S.-W. Cheong, and A. S. Cooper, Phys. Rev. Lett. 71, 2461 (1993); J. M. Tranquada, D. J. Buttrey, V. Sachan, and J. E. Lorenzo, Phys. Rev. Lett. 73, 1003 (1994); J. M. Tranquada, J. E. Lorenzo, D. J. Buttrey, and V. Sachan, Phys. Rev. B 52, 3581 (1995); V. Sachan et al., Phys. Rev. B 51, 12742 (1995).

[2] J. Tranquada et al, Nature 375, 561 (1995): Phys. Rev. B 54, 7489 (1996). J. M. Tranquada, J. D. Axe, N. Ichikawa, A. R. Moodenbaugh, Y. Nakamura, and S. Uchida, preprint.

[3] S-W. Cheong, et al., Phys. Rev. Lett. 67, 1791 (1991). T.E. Mason, et al., Phys. Rev. Lett. 68, 1414 (1992). T. R. Thurston, et al., Phys. Rev. B 46, 9128 (1992). K. Yamada et al, Sendai preprint (1996).

[4] L. D. Landau and E. M. Lifschitz, Statistical Physics (Addison-Wesley, Reading, 1958).

[5] V. J. Emery, S. A. Kivelson, and H.-Q. Lin, Phys. Rev. Lett. 64, 475 (1990); S. A. Kivelson, V. J. Emery, and H-Q. Lin, Phys. Rev. B 42, 6523 (1990); V. J. Emery and S. A. Kivelson, Physica C 209, 597 (1993). S. A. Kivelson and V. J. Emery in Strongly Correlated Electronic Materials: The Los Alamos Symposium 1993 edited by K. S. Bedell et al. (Addison Wesley, Redwood City, 1994) p. 619 .

[6] U. Löw, V. J. Emery, K. Fabricius, and S. A. Kivelson, Phys. Rev. Lett. 72, 1918 (1994).

[7] L. Chayes, V. J. Emery, S. A. Kivelson, Z. Nussinov, and J. Tarjus, Physica A225, 129 (1996).

[8] J. Zaanen and O. Gunnarson, Phys. Rev. B 40, 7391 (1989); D. Poilblanc and T. M. Rice, Phys. Rev. B 39, 9749 (1989); H. Schultz, Phys. Rev. Lett. 64, 1445 (1990). J. Phys. (Paris) 50, 2833 (1989); J. A. Vergés et al., Phys. Rev. B 43, 6099 (1991); M. Inui and P. Littlewood, Phys. Rev. B 44, 4415 (1991); T. Giamarchi and C. Lhuillier, Phys. Rev. B 42, 10641 (1990); G. An and G. M. J. van Leeuwen, Phys. Rev. B44, 9410 (1991);

[9] B. I. Shraiman and E. D. Siggia, Phys. Rev. Lett. 61, 467 (1988) and Phys. Rev. B 46, 8305 (1992); C. Zhou and H. J. Schulz, Phys. Rev. B 52, 11557 (1995).

[10] S. A. Kivelson and V. J. Emery, Synthetic Metals, 80, 151 (1996).

[11] P. Bak and V. J. Emery, Phys. Rev. Lett. 36, 978 (1976); W. L. McMillan, Phys. Rev. B 14, 1496 (1976).

[12] V. J. Emery and S. A. Kivelson, Phys. Rev. Lett. 74, 3253 (1995).

[13] G. Gomez-Santos, J. D. Joannopoulos, and J. W. Negele, Phys. Rev. B 39, 4435 (1989); E. Manousakis and R. Salvador, Phys. Rev. Lett. 60, 840 (1988).

[14] P. A. Lee and T. M. Rice, Phys. Rev. B 19, 3970 (1979). 